\title{
Positive Solutions for a Nonhomogeneous Kirchhoff Equation with the Asymptotical Nonlinearity in $R^{3}$
}

\author{
Ling Ding, ${ }^{1}$ Lin Li, ${ }^{2}$ and Jin-Ling Zhang ${ }^{1}$ \\ ${ }^{1}$ School of Mathematics and Computer Science, Hubei University of Arts and Science, Hubei 441053, China \\ ${ }^{2}$ School of Mathematics and Statistics, Southwest University, Chongqing 400715, China \\ Correspondence should be addressed to Ling Ding; dingling1975@qq.com
}

Received 19 July 2013; Revised 20 December 2013; Accepted 22 December 2013; Published 25 February 2014

Academic Editor: Elena Litsyn

Copyright (c) 2014 Ling Ding et al. This is an open access article distributed under the Creative Commons Attribution License, which permits unrestricted use, distribution, and reproduction in any medium, provided the original work is properly cited.

We study the following nonhomogeneous Kirchhoff equation: $-\left(a+b \int_{R^{3}}|\nabla u|^{2} d x\right) \Delta u+u=k(x) f(u)+h(x), x \in R^{3}, u \in$ $H^{1}\left(R^{3}\right), u>0, x \in R^{3}$, where $f$ is asymptotically linear with respect to $t$ at infinity. Under appropriate assumptions on $k, f$, and $h$, existence of two positive solutions is proved by using the Ekeland's variational principle and the Mountain Pass Theorem in critical point theory.

\section{Introduction and Main Results}

In this paper, we consider the following nonhomogeneous Kirchhoff equation:

$$
\begin{array}{r}
-\left(a+b \int_{R^{3}}|\nabla u|^{2} d x\right) \Delta u+u=k(x) f(u)+h(x), \\
x \in R^{3}, \\
u \in H^{1}\left(R^{3}\right), \quad u>0, x \in R^{3},
\end{array}
$$

where constants $a, b>0$, and functions $k, f$ and $h$ satisfy the following conditions: $k$ is a positive bounded condition, $f \in$ $C\left(R, R^{+}\right), f(t) \equiv 0$ if $t<0$ and $h \in L^{2}\left(R^{3}\right), h \geq 0$. Note that, with $a=1, b=0$, and $R^{3}$ replaced by $R^{N}$, problem (1) reduces to

$$
-\Delta u+u=k(x) f(u)+h(x) \quad \text { in } R^{N},
$$

which can be looked at as a generalization of the well known Schrödinger equation.

When $\Omega$ is a smooth bounded domain in $R^{N}$, the problem

$$
\begin{gathered}
-\left(a+b \int_{\Omega}|\nabla u|^{2} d x\right) \Delta u=g(x, u), \quad x \in \Omega, \\
u=0, \quad x \in \partial \Omega,
\end{gathered}
$$

is related to the stationary analogue of the Kirchhoff equation which was proposed by Kirchhoff in 1883 (see [1]) as a generalization of the well known d'Alembert's wave equation

$$
\rho \frac{\partial^{2} u}{\partial t^{2}}-\left(\frac{P_{0}}{h}+\frac{E}{2 L} \int_{0}^{L}\left|\frac{\partial u}{\partial x}\right|^{2} d x\right) \frac{\partial^{2} u}{\partial x^{2}}=g(x, u)
$$

for free vibrations of elastic strings. Kirchhoff's model takes into account the changes in length of the string produced by transverse vibrations. Here, $L$ is the length of the string, $h$ is the area of the cross section, $E$ is the Young modulus of the material, $\rho$ is the mass density, and $P_{0}$ is the initial tension. Moreover, Kirchhoff's type problems also model several physical systems and biological systems and there are many interesting results for problem (3) which can be found in [2-8] and the references therein.

Some interesting studies for Kirchhoff-type problem (3) in a bounded domain $\Omega$ of $R^{N}$ by variational methods can be found in $[2,9-22]$. Very recently, some authors had studied the Kirchhoff equation on the whole space $R^{N}$ and obtained the existence of multiple solutions (see [23-31]). In the same spirit of [24-26, 28-31], we study a nonhomogeneous Kirchhoff equation (1) on the whole space $R^{3}$. Especially, inspired by the paper [32,33], we consider the asymptotically linear nonlinearity at infinity of problem (1). For the nonhomogeneous Kirchhoff problem, Chen and $\mathrm{Li}$ in 
[23] study it under the condition of superlinear nonlinearity at infinity. In [33], Wang and Zhou study the existence of two positive solutions for a nonhomogeneous elliptic equation ((1) with $a=1$ and $b=0$ ). In [32], Sun et al. study the existence of a ground state solution for some nonautonomous Schrödinger-Poisson systems involving the asymptotically linear nonlinearity at infinity without the nonhomogeneous term. But we will study the existence of two positive solutions for Kirchhoff-type problem (1) with $a, b>0$, the asymptotically linear nonlinearity at infinity and the nonhomogeneous term. So, we can not obtain the existence of a ground state solution for Kirchhoff-type problem (1) and the compactness result as in [32] because of the nonhomogeneous term, and we cannot easily obtain the compactness result as in [33] due to the nonlocal term (or $b \neq 0$ ). To our best knowledge, little has been done for nonhomogeneous Kirchhoff problems with respect to the asymptotically linear nonlinearity at infinity.

Before stating our main results, we give some notations. For any $1 \leq q \leq+\infty$, we denote by $\|\cdot\|_{q}$ the usual norm of the Lebesgue space $L^{q}\left(R^{3}\right)$. Define the function space

$$
H^{1}\left(R^{3}\right):=\left\{u \in L^{2}\left(R^{3}\right): \nabla u \in L^{2}\left(R^{3}\right)\right\}
$$

with the product and equivalent norm

$$
\begin{aligned}
& (u, v)=\int_{R^{3}}(a \nabla u \cdot \nabla v+u v) d x \\
& \|u\|:=\left(\int_{R^{3}}\left(a|\nabla u|^{2}+|u|^{2}\right) d x\right)^{1 / 2} .
\end{aligned}
$$

Define the function space

$$
D^{1,2}\left(R^{3}\right):=\left\{u \in L^{6}\left(R^{3}\right): \nabla u \in L^{2}\left(R^{3}\right)\right\}
$$

with the standard product and norm

$$
(u, v)=\int_{R^{3}} \nabla u \cdot \nabla v d x, \quad\|u\|_{D}:=\left(\int_{R^{3}}|\nabla u|^{2} d x\right)^{1 / 2} .
$$

Recall that the Sobolev's inequality with the best constant is

$$
\|v\|_{6} \leq S\|v\| \text {. }
$$

Moreover, problem (1) has a variational structure. Indeed the corresponding action functional $I: H^{1}\left(R^{3}\right) \rightarrow R$ of $(1)$ is defined by

$$
\begin{aligned}
I(u)= & \frac{a}{2} \int_{R^{3}}|\nabla u|^{2} d x+\frac{b}{4}\left(\int_{R^{3}}|\nabla u|^{2} d x\right)^{2}+\frac{1}{2} \int_{R^{3}}|u|^{2} d x \\
& -\int_{R^{3}} k(x) F(u) d x-\int_{R^{3}} h(x) u d x .
\end{aligned}
$$

By Lemma 2.1 in [24] or Lemma 1 in [25], the functional $I$ is $C^{1}\left(H^{1}\left(R^{3}\right), R\right)$ with the derivative given by

$$
\begin{aligned}
\left\langle I^{\prime}(u), u\right\rangle= & \left(a+b \int_{R^{3}}|\nabla u|^{2} d x\right) \int_{R^{3}} \nabla u \cdot \nabla v d x+\int_{R^{3}} u v d x \\
& -\int_{R^{3}} k(x) f(u) u d x-\int_{R^{3}} h(x) v d x .
\end{aligned}
$$

Hence, if $u \in H^{1}\left(R^{3}\right)$ is a nonzero critical point of $I$, then it is also a nonnegative solution of (1). In fact, by $f(t) \equiv 0$ if $t<0$ and $h \geq 0$, we have $\left\langle I^{\prime}(u), u^{-}\right\rangle=-\left(a+b\|u\|^{2}\right) \int_{R^{3}}\left|\nabla u^{-}\right|^{2} d x-$ $\int_{R^{3}}\left(u^{-}\right)^{2} d x-\int_{R^{3}} k(x) f(u) u^{-} d x-\int_{R^{3}} h(x) u^{-} d x=0$, where $u^{-}=\max \{-u, 0\}$. This yields that $u^{-}=0$; then $u=u^{+}-u^{-}=$ $u^{+} \geq 0$, where $u^{+}=\max \{u, 0\}$. By the maximum principle, the nonzero critical point of $I$ is the positive solution for problem (1).

Here is the main result of this paper.

Theorem 1. Suppose that $h \in L^{2}\left(R^{3}\right), h \geq 0$, and the following conditions hold.

(f1) $f \in C\left(R, R^{+}\right), f(0)=0$, and $f(t) \equiv 0$ for $t<0$.

(f2) $\lim _{t \rightarrow 0}(f(t) / t)=0$.

(f3) $\lim _{t \rightarrow+\infty}(f(t) / t)=l<+\infty$.

(k1) $k(x)$ is a positive continuous function and there exists $R_{0}>0$ such that

$$
\sup \left\{\frac{f(t)}{t}: t>0\right\}<\inf \left\{\frac{1}{k(x)}:|x| \geq R_{0}\right\} \text {. }
$$

(k2) Let

$$
\begin{gathered}
l_{0}>\mu^{*}:=\inf \left\{\int_{R^{3}}\left(a|\nabla u|^{2}+u^{2}\right) d x: u \in H^{1}\left(R^{3}\right),\right. \\
\left.\int_{R^{3}} k(x) F(u) d x \geq \frac{b}{2} l^{2}\right\}
\end{gathered}
$$

hold, where $l_{0}=\min \left\{l,(b / 2) l^{2}\right\}, F(t)=\int_{0}^{t} f(s) d s$.

Then problem (1) has at least two positive solutions $u_{0}, u \in$ $H^{1}\left(R^{3}\right)$ satisfying $I\left(u_{0}\right)<0$ and $I(u)>0$ if $\|h\|_{2}<m$ for some small $m>0$.

Remark 2. It is not difficult to find some functions $k, f$ satisfying conditions of Theorem 1 . For example, for any $R_{0}>$ 0 , let

$$
f(t)= \begin{cases}\frac{R_{0} t^{2}}{1+t}, & \text { if } t \geq 0 \\ 0, & \text { if } t<0\end{cases}
$$

Clearly, $f$ satisfies (f1)-(f3) with $l=R_{0}$. Moreover, $F(t)=$ $R_{0}\left((1 / 2) t^{2}-t+\ln (1+t)\right)$ and $\sup \{f(t) / t: t>0\}=R_{0}$. Taking a positive continuous function $k(x)$,

$$
k(x)= \begin{cases}\frac{C_{0}}{1+|x|}, & \text { if }|x| \leq \frac{R_{0}}{2}, \\ \frac{1}{1+\left|R_{0}\right|}, & \text { if }|x| \geq R_{0},\end{cases}
$$

where $C_{0}=3 M^{3}\left(1+R_{0} / 2\right) / 4 \pi(\ln 2-1 / 2)$ for some $M>0$. Note that

$$
\begin{aligned}
\inf \left\{\frac{1}{k(x)}:|x| \geq R_{0}\right\} & =1+R_{0}>R_{0} \\
& =\sup \left\{\frac{f(t)}{t}: t>0\right\} ;
\end{aligned}
$$


then (k1) holds. To verify the condition (k2), we have to choose some special $R_{0}>0$. For any $R>0$, taking $\psi \epsilon$ $C_{0}^{\infty}\left(R^{3},[0,1]\right)$ such that $\psi(x)=1$ if $|x| \leq R, \psi(x)=0$ if $|x| \geq 2 R$ and $|\nabla \psi(x)| \leq C / \sqrt{a} R$ for all $x \in R^{3}$, where $C>0$ is an arbitrary constant independent of $x$. Then, for any $R_{0}>2 R$, we have

$$
\begin{aligned}
\int_{R^{3}} k(x) F(\psi) d x & \geq \int_{|x| \leq R} k(x) F(\psi) d x \\
& \geq \frac{R_{0} C_{0}}{1+R}\left(\ln 2-\frac{1}{2}\right)\left|B_{R}(0)\right| \\
& \geq \frac{3 M^{3}\left(1+R_{0} / 2\right)}{4 \pi(\ln 2-1 / 2)} \frac{4 \pi R^{3} R_{0}}{3\left(1+R_{0} / 2\right)} \\
& \times\left(\ln 2-\frac{1}{2}\right) \\
& =M^{3} R_{0} R^{3}, \\
\int_{R^{3}}\left(a|\nabla \psi|^{2}+|\psi|^{2}\right) d x & \leq \int_{|x| \leq 2 R} \frac{C^{2}}{R^{2}} d x+\int_{|x| \leq 2 R} d x \\
\leq & \left(1+\frac{C^{2}}{R^{2}}\right) \frac{32 \pi}{3} R^{3} \\
\leq & \frac{32 \pi}{3} R\left(C^{2}+R^{2}\right) .
\end{aligned}
$$

Taking $R_{0}=l=1, R=(1 / M) R_{0} \sqrt[3]{b / 2}=(1 / M) \sqrt[3]{b / 2}$, where $M$ is large enough such that $2 R<R_{0} / 4,40 \pi / 3 M^{3}<1$, and $\left(40 \pi / 3 M^{3}\right)(b / 2)<1$. Let $C=(1 / 4 M) \sqrt[3]{b / 2}$. Then, we obtain that $\int_{R^{3}} k(x) F(\psi) d x \geq b R_{0} / 2=b l^{2} / 2$. Moreover, in view of the definition of $\mu^{*}$ and (18), one has

$$
\begin{aligned}
\mu^{*} & \leq \int_{R^{3}}\left(a|\nabla \psi|^{2}+|\psi|^{2}\right) d x \\
& \leq \frac{32 \pi}{3} R\left(C^{2}+R^{2}\right)<\frac{40 \pi}{3 M^{3}} \frac{b}{2}<l_{0} .
\end{aligned}
$$

So, condition (k2) holds. In particular, the condition (k1) and above examples can also be found in [34] in which the asymptotically linear term $k(x) f(u)$ satisfying $(\mathrm{k} 1)$ appeared first.

Remark 3. If $h \equiv 0$, we know that problem (1) has a positive ground state solution by using the method in [32] and a trivial solution $(u(x) \equiv 0)$. If $h \equiv 0$, a trivial solution $(u(x) \equiv 0)$ is replaced by the local minimum solution by Theorem 1 . Note that the local minimal solution exists due to the homogeneous term which is looked at as a small perturbation because $\|h\|_{2}<m$ for small $m$.

In order to obtain our results, we have to overcome various difficulties. Since the embedding of $H^{1}\left(R^{3}\right)$ into $L^{p}\left(R^{3}\right)$, $p \in[2,6]$, is not compact, condition (k1) and (k2) are crucial to obtain the boundedness of Cerami sequence. Furthermore, in order to recover the compactness, we establish a compactness result $\int_{|x| \geq R}\left(\left|\nabla u_{n}\right|^{2}+\left|u_{n}\right|^{2}\right) d x \leq \varepsilon$ which is similar to [32] but different from the one in [24-26, 28-31]. In fact, this difficulty can be avoided, when problems are considered, restricting $I$ to the subspace of $H^{1}\left(R^{3}\right)$ consisting of radially symmetric functions [23, 24, 29] and constraint potential functions $[25,30]$, or when one is looking for semiclassical states [28], by using perturbation methods or a reduction to a finite dimension by the projections method. Third, it is not difficult to find that every (PS) sequence is bounded because a variant of Ambrosetti-Rabinowitz condition is satisfied (see $[23,25,31])$. However, for the asymptotically linear case, we have to find another method to verify the boundedness of (PS) sequence.

This paper is organized as follows. In Section 2, we manage to give proofs of Theorem 1 . In the following discussion, we denote various positive constants as $C$ or $C_{i}(i=$ $1,2,3, \ldots)$ for convenience.

\section{Proof of Main Result}

In this section, we prove that problem (1) has a mountain pass type solution and a local minimum solution with $h \not \equiv 0$. For this purpose, we use a variant version of Mountain Pass Theorem [35], which allows us to find a so-called Cerami type (PS) sequence (Cerami sequence, in short). The properties of this kind of Cerami sequence sequences are very helpful in showing its boundedness in the asymptotical cases. The following lemmas will show that $I$ has the so-called mountain pass geometry.

Lemma 4. Suppose that $h \in L^{2}\left(R^{3}\right), h \geq 0,(f 1)-(f 3)$, and ( $\left.k 1\right)$ hold. Then there exist $\rho, \alpha, m>0$ such that $\left.I(u)\right|_{\|u\|=\rho} \geq \alpha>0$ for $\|h\|_{2}<m$.

Proof. For any $\varepsilon>0$, it follows from (f1)-(f3) that there exists $C_{\varepsilon}>0$ such that

$$
|f(t)| \leq \varepsilon|t|+C_{\varepsilon}|t|^{5} \quad \forall t \in R .
$$

Therefore, we have

$$
|F(t)| \leq \frac{1}{2} \varepsilon|t|^{2}+\frac{C_{\varepsilon}}{6}|t|^{6} \quad \forall t \in R .
$$

Furthermore, by (f1)-(f3) and (k1), there exists $C_{1}>0$ such that

$$
k(x) \leq C_{1} \quad \forall x \in R^{3}
$$

According to (21), (22), and the Sobolev inequality, we deduce that

$$
\begin{aligned}
\left|\int_{R^{3}} k(x) F(u) d x\right| & \leq \frac{\varepsilon C_{1}}{2} \int_{R^{3}}|u|^{2} d x+\frac{C_{1} C_{\varepsilon}}{6} \int_{R^{3}}|u|^{6} d x \\
& \leq \frac{\varepsilon C_{1}}{2}\|u\|^{2}+C_{2}\|u\|^{6},
\end{aligned}
$$


where $C_{2}=C_{1} C_{\varepsilon} S^{6} / 6$. By $b>0, h \in L^{2}\left(R^{3}\right)$, and the Hölder inequality, one has

$$
\begin{aligned}
I(u)= & \frac{a}{2} \int_{R^{3}}|\nabla u|^{2} d x+\frac{b}{4}\left(\int_{R^{3}}|\nabla u|^{2} d x\right)^{2}+\frac{1}{2} \int_{R^{3}}|u|^{2} d x \\
& -\int_{R^{3}} k(x) F(u) d x-\int_{R^{3}} h(x) u d x \\
\geq & \frac{a}{2} \int_{R^{3}}|\nabla u|^{2} d x+\frac{1}{2} \int_{R^{3}}|u|^{2} d x-\frac{\varepsilon C_{1}}{2}\|u\|^{2} \\
& -C_{2}\|u\|^{6}-\|h\|_{2}\|u\|_{2} \\
\geq & \frac{1}{2}\|u\|^{2}-\frac{\varepsilon C_{1}}{2}\|u\|^{2}-C_{2}\|u\|^{6}-\|h\|_{2}\|u\| \\
\geq & \|u\|\left(\frac{1-\varepsilon C_{1}}{2}\|u\|-C_{2}\|u\|^{5}-\|h\|_{2}\right) .
\end{aligned}
$$

Taking $\varepsilon=1 / 2 C_{1}$ and setting $g(t)=(1 / 4) t-C_{2} t^{5}$ for $t \geq 0$, we see there exists $\rho=\left(1 / 20 C_{2}\right)^{1 / 4}$ such that $\max _{t \geq 0} g(t)=$ $g(\rho):=m>0$. Then it follows from (24) that there exists $\alpha>0$ such that $\left.I(u)\right|_{\|u\|=\rho} \geq \alpha>0$ for $\|h\|_{2}<m$. Of course, $\rho, m$ can be chosen small enough; we can obtain the same result: there exists $\alpha>0$ such that $\left.I(u)\right|_{\|u\|=\rho} \geq \alpha>0$ for $\|h\|_{2}<m$.

Lemma 5. Suppose that $h \in L^{2}\left(R^{3}\right), h \geq 0$, (f1)-(f3), and (k1)(k2) hold. Then there exists $v \in H^{1}\left(R^{3}\right)$ with $\|v\|>\rho$, $\rho$ is given by Lemma 4, such that $I(v)<0$.

Proof. By (k2) and $h \geq 0$, in view of the definition of $\mu^{*}$ and $l_{0}>\mu^{*}$ with $l_{0}=\min \left\{l,(b / 2) l^{2}\right\}$, there is a nonnegative function $v \in H^{1}\left(R^{3}\right)$ such that

$$
\int_{R^{3}} k(x) F(v) d x \geq \frac{b}{2} l^{2}, \quad \int_{R^{3}} h(x) v d x \geq 0,
$$

and $\mu^{*} \leq\|v\|^{2}<l_{0}$. Then, we have

$$
\begin{aligned}
I(v)= & \frac{a}{2} \int_{R^{3}}|\nabla v|^{2} d x+\frac{b}{4}\left(\int_{R^{3}}|\nabla v|^{2} d x\right)^{2}+\frac{1}{2} \int_{R^{3}}|v|^{2} d x \\
& -\int_{R^{3}} k(x) F(v) d x-\int_{R^{3}} h(x) v d x \\
\leq & \frac{1}{2}\|v\|^{2}+\frac{b}{4}\|v\|^{4}-\frac{b}{2} l^{2} \\
\leq & \frac{1}{2}\|v\|^{2}-\frac{b}{4} l^{2} \\
& <0 .
\end{aligned}
$$

Choosing $\rho>0$ small enough in Lemma 4 such that $\|v\|>\rho$, then this Lemma is proved.

From Lemmas 4 and 5 and Mountain Pass Lemma in [35], there is a Cerami sequence $\left\{u_{n}\right\} \subset H^{1}\left(R^{3}\right)$ such that

$$
\begin{aligned}
& \left\|I^{\prime}\left(u_{n}\right)\right\|_{H^{-1}}\left(1+\left\|u_{n}\right\|\right) \longrightarrow 0, \\
& I\left(u_{n}\right) \longrightarrow c \text { as } n \longrightarrow \infty,
\end{aligned}
$$

where $H^{-1}$ denotes the dual space of $H^{1}\left(R^{3}\right)$. In the following Lemmas 6 and 7, we shall prove that $I$ satisfies the Cerami condition, that is; the Cerami sequence $\left\{u_{n}\right\}$ has a convergence subsequence.

Lemma 6. Suppose that $h \in L^{2}\left(R^{3}\right), h \geq 0,(f 1)-(f 3)$, and ( $\left.k 1\right)$ hold. Then $\left\{u_{n}\right\}$ defined in (27) is bounded in $H^{1}\left(R^{3}\right)$.

Proof. By contradiction, let $\left\|u_{n}\right\| \rightarrow \infty$. Define $w_{n}=$ $u_{n}\left\|u_{n}\right\|^{-1}$. Clearly, $\left\{w_{n}\right\}$ is bounded in $H^{1}\left(R^{3}\right)$ and there is a $w \in H^{1}\left(R^{3}\right)$ such that, up to a sequence,

$$
\begin{gathered}
w_{n} \longrightarrow w \quad \text { weakly in } H^{1}\left(R^{3}\right), \\
w_{n} \longrightarrow w \quad \text { a.e. in } R^{3}, \\
w_{n} \longrightarrow w \quad \text { strongly in } L_{l o c}^{2}\left(R^{3}\right)
\end{gathered}
$$

as $n \rightarrow \infty$.

Firstly, we claim that $w$ is nontrivial; that is, $w$ \# 0 . Otherwise, if $w \equiv 0$, the Sobolev embedding implies that $w_{n} \rightarrow 0$ strongly in $L^{2}\left(B_{R_{0}}\right) ; R_{0}$ is given by (k1). By (f1)-(f3), there exists $C_{3}>0$ such that

$$
\frac{f(t)}{t} \leq C_{3} \quad \forall t \in R .
$$

Then, for all $n \in N$, we have

$$
0 \leq \int_{|x|<R_{0}} k(x) \frac{f\left(u_{n}\right)}{u_{n}} w_{n}^{2} d x \leq C_{3}\|k\|_{\infty} \int_{|x|<R_{0}} w_{n}^{2} d x \longrightarrow 0 .
$$

This yields

$$
\lim _{n \rightarrow \infty} \int_{|x|<R_{0}} k(x) \frac{f\left(u_{n}\right)}{u_{n}} w_{n}^{2} d x=0 .
$$

Furthermore, by $(\mathrm{k} 1)$, there exists a constant $\theta \in(0,1)$ such that

$$
\sup \left\{\frac{f(t)}{t}: t>0\right\} \leq \theta \inf \left\{\frac{1}{k(x)}:|x| \geq R_{0}\right\} .
$$

Then, for all $n \in N$, we have

$$
\int_{|x| \geq R_{0}} k(x) \frac{f\left(u_{n}\right)}{u_{n}} w_{n}^{2} d x \leq \theta \int_{|x| \geq R_{0}} w_{n}^{2} d x \leq \theta\|w\|^{2}=\theta<1 .
$$

Combining (31) and (33), we obtain

$$
\limsup _{n \rightarrow \infty} \int_{R^{3}} k(x) \frac{f\left(u_{n}\right)}{u_{n}} w_{n}^{2} d x<1 .
$$

By (27), we get

$$
\begin{aligned}
0 & \leq\left|\left\langle I^{\prime}\left(u_{n}\right), u_{n}\right\rangle\right| \leq\left\|I^{\prime}\left(u_{n}\right)\right\|_{H^{-1}}\left\|u_{n}\right\| \\
& \leq\left\|I^{\prime}\left(u_{n}\right)\right\|_{H^{-1}}\left(1+\left\|u_{n}\right\|\right) \longrightarrow 0
\end{aligned}
$$


as $n \rightarrow \infty$. Together with $\left\|u_{n}\right\| \rightarrow \infty$ as $n \rightarrow \infty$, it follows that

$$
\frac{\left\langle I^{\prime}\left(u_{n}\right), u_{n}\right\rangle}{\left\|u_{n}\right\|^{2}}=o(1)
$$

Together with $b>0$, we have

$$
\begin{aligned}
o(1)=\frac{1}{\left\|u_{n}\right\|^{2}}\left(a \int_{R^{3}}\left|\nabla u_{n}\right|^{2} d x+\int_{R^{3}}\left|u_{n}\right|^{2} d x\right. \\
\quad+b\left(\int_{R^{3}}\left|\nabla u_{n}\right|^{2} d x\right)^{2} \\
\left.\quad-\int_{R^{3}} k(x) f\left(u_{n}\right) u_{n} d x-\int_{R^{3}} h(x) u_{n} d x\right) \\
\geq\left\|w_{n}\right\|^{2}-\int_{R^{3}} k(x) \frac{f\left(u_{n}\right)}{u_{n}} w_{n}^{2} d x \\
\geq 1-\int_{R^{3}} k(x) \frac{f\left(u_{n}\right)}{u_{n}} w_{n}^{2} d x
\end{aligned}
$$

where, and in what follows, $o(1)$ denotes a quantity which goes to zero as $n \rightarrow \infty$. Therefore, we deduce that

$$
\int_{R^{3}} k(x) \frac{f\left(u_{n}\right)}{u_{n}} w_{n}^{2} d x+o(1) \geq 1
$$

which contradicts (34). So, $w \neq \equiv 0$.

Furthermore, because $\left\|u_{n}\right\| \rightarrow \infty$ as $n \rightarrow \infty$, it follows from (35) that

$$
\frac{\left\langle I^{\prime}\left(u_{n}\right), u_{n}\right\rangle}{\left\|u_{n}\right\|^{4}}=o(1)
$$

that is,

$$
o(1)=\frac{b\left(\int_{R^{3}}\left|\nabla u_{n}\right|^{2} d x\right)^{2}}{\left\|u_{n}\right\|^{4}}-\frac{1}{\left\|u_{n}\right\|^{2}} \int_{R^{3}} k(x) \frac{f\left(u_{n}\right)}{u_{n}} w_{n}^{2} d x
$$

Together with (22), (29), and $b>0$, one has

$$
\begin{aligned}
& \frac{\left(\int_{R^{3}}\left|\nabla u_{n}\right|^{2} d x\right)^{2}}{\left\|u_{n}\right\|^{4}} \\
& =\frac{\left(\int_{R^{3}}\left(\left|\nabla u_{n}\right|^{2}+\left|u_{n}\right|^{2}\right) d x-\int_{R^{3}}\left|u_{n}\right|^{2} d x\right)^{2}}{\left\|u_{n}\right\|^{4}}=o(1) .
\end{aligned}
$$

This yields

$$
\begin{aligned}
& \frac{\left(\int_{R^{3}}\left(\left|\nabla u_{n}\right|^{2}+\left|u_{n}\right|^{2}\right) d x\right)^{2}}{\left\|u_{n}\right\|^{4}} \\
& -\frac{2 \int_{R^{3}}\left(\left|\nabla u_{n}\right|^{2}+\left|u_{n}\right|^{2}\right) d x \int_{R^{3}}\left|u_{n}\right|^{2} d x}{\left\|u_{n}\right\|^{4}} \\
& +\frac{\left(\int_{R^{3}}\left|u_{n}\right|^{2} d x\right)^{2}}{\left\|u_{n}\right\|^{4}}=o(1) .
\end{aligned}
$$

This means

$$
\begin{aligned}
1-2 \int_{R^{3}}\left|w_{n}\right|^{2} d x+\left(\int_{R^{3}}\left|w_{n}\right|^{2} d x\right)^{2} & =\left(1-\int_{R^{3}}\left|w_{n}\right|^{2} d x\right)^{2} \\
& =o(1)
\end{aligned}
$$

Therefore, we have

$$
\int_{R^{3}}\left|w_{n}\right|^{2} d x \longrightarrow 1 \text { as } n \longrightarrow \infty
$$

By $\left\|w_{n}\right\|=1$, we get $\int_{R^{3}}\left|\nabla w_{n}\right|^{2} d x \rightarrow 0$ as $n \rightarrow \infty$; thus $w_{n} \rightarrow 0$ strongly in $D^{1,2}\left(R^{3}\right)$; therefore, $w_{n} \rightarrow 0$ weakly in $D^{1,2}\left(R^{3}\right)$. Since $w_{n} \rightarrow w$ weakly in $H^{1}\left(R^{3}\right)$; we have $w_{n} \rightarrow w$ weakly in $D^{1,2}\left(R^{3}\right)$. By the uniqueness of the weak limitation, we have $w=0$ which contradicts $w \neq 0$. Therefore, the Cerami sequence $\left\{u_{n}\right\}$ is bounded in $H^{1}\left(R^{3}\right)$.

Lemma 7. Suppose that $h \in L^{2}\left(R^{3}\right), h \geq 0,(f 1)-(f 3)$, and $(k 1)$ hold. Then for any $\varepsilon>0$, there exist $R(\varepsilon)>R_{0}$ and $n(\varepsilon)>0$ such that $\left\{u_{n}\right\}$ defined in (27) satisfies $\int_{|x| \geq R}\left(\left|\nabla u_{n}\right|^{2}+\left|u_{n}\right|^{2}\right) d x \leq$ $\varepsilon$ for $n>n(\varepsilon)$ and $R \geq R(\varepsilon)$.

Proof. Let $\xi_{R}: R^{3} \rightarrow[0,1]$ be a smooth function such that

$$
\xi_{R}(x)= \begin{cases}0, & 0 \leq|x| \leq \frac{R}{2} \\ 1, & |x| \geq R\end{cases}
$$

Moreover, there exists a constant $C_{4}$ independent of $R$ such that

$$
\left|\nabla \xi_{R}(x)\right| \leq \frac{C_{4}}{R} \quad \forall x \in R^{3}
$$


Then, for all $n \in N$ and $R \geq R_{0}$, by (45), (46), and the Hölder inequality, we have

$$
\begin{aligned}
& \int_{R^{3}}\left|\nabla\left(u_{n} \xi_{R}\right)\right|^{2} d x \leq \int_{R^{3}}\left|u_{n}\right|^{2}\left|\nabla \xi_{R}\right|^{2} d x+\int_{R^{3}}\left|\nabla u_{n}\right|^{2}\left|\xi_{R}\right|^{2} d x \\
& +2 \int_{R^{3}}\left|u_{n}\right|\left|\xi_{R}\right|\left|\nabla u_{n}\right|\left|\nabla \xi_{R}\right| d x \\
& \leq \int_{R / 2<|x|<R}\left|\nabla u_{n}\right|^{2} d x+\int_{|x|>R}\left|\nabla u_{n}\right|^{2} d x \\
& +\frac{C_{4}^{2}}{R^{2}} \int_{R^{3}}\left|u_{n}\right|^{2} d x \\
& +2\left(\int_{R^{3}}\left|\nabla u_{n}\right|^{2}\left|\xi_{R}^{2}\right| d x\right)^{1 / 2} \\
& \times\left(\int_{R^{3}}\left|u_{n}\right|^{2}\left|\nabla \xi_{R}\right|^{2} d x\right)^{1 / 2} \\
& \leq \int_{R / 2<|x|<R}\left|\nabla u_{n}\right|^{2} d x+\int_{|x|>R}\left|\nabla u_{n}\right|^{2} d x \\
& +\frac{C_{4}^{2}}{R^{2}} \int_{R^{3}}\left|u_{n}\right|^{2} d x \\
& +2\left(\int_{R / 2<|x|<R}\left|\nabla u_{n}\right|^{2} d x\right. \\
& \left.+\int_{|x|>R}\left|\nabla u_{n}\right|^{2} d x\right)^{1 / 2} \\
& \times\left(\frac{C_{4}^{2}}{R^{2}} \int_{R^{3}}\left|u_{n}\right|^{2} d x\right)^{1 / 2} \\
& \leq\left(2+\frac{C_{4}^{2}}{R^{2}}+\frac{2 \sqrt{2} C_{4}}{R}\right)\left\|u_{n}\right\|^{2} \\
& \leq\left(2+\frac{C_{4}^{2}}{R_{0}^{2}}+\frac{2 \sqrt{2} C_{4}}{R_{0}}\right)\left\|u_{n}\right\|^{2} .
\end{aligned}
$$

This implies that

$$
\left\|u_{n} \xi_{R}\right\| \leq C_{5}\left\|u_{n}\right\|
$$

for all $n \in N$ and $R \geq R_{0}$, where $C_{5}=$ $\max \left\{\left(3+\left(C_{4}^{2} / R_{0}^{2}\right)+2 \sqrt{2} C_{4} / R_{0}\right)^{1 / 2}, 1\right\}$. From Lemma 6, we know that $\left\{u_{n}\right\}$ is bounded in $H^{1}\left(R^{3}\right)$. Together with (27), we obtain that $I^{\prime}\left(u_{n}\right) \rightarrow 0$ in $H^{-1}\left(R^{3}\right)$. Moreover, by (48), for $\varepsilon>0$, there exists $n(\varepsilon)>0$ such that

$$
\left\langle I^{\prime}\left(u_{n}\right), \xi_{R} u_{n}\right\rangle \leq C_{5}\left\|I^{\prime}\left(u_{n}\right)\right\|_{H^{-1}\left(R^{3}\right)}\left\|u_{n}\right\| \leq \frac{\varepsilon}{4}
$$

for $n>n(\varepsilon)$ and $R>R_{0}$. Note that

$$
\begin{aligned}
\left\langle I^{\prime}\left(u_{n}\right), \xi_{R} u_{n}\right\rangle= & \left(a+b \int_{R^{3}}\left|\nabla u_{n}\right|^{2} d x\right) \\
& \times \int_{R^{3}}\left|\nabla u_{n}\right|^{2} \xi_{R} d x+\int_{R^{3}}\left|u_{n}\right|^{2} \xi_{R} d x \\
& +\left(a+b \int_{R^{3}}\left|\nabla u_{n}\right|^{2} d x\right) \\
& \times \int_{R^{3}} u_{n} \nabla u_{n} \cdot \nabla \xi_{R} d x \\
& -\int_{R^{3}} k(x) f\left(u_{n}\right) u_{n} \xi_{R} d x \\
& -\int_{R^{3}} h(x) u_{n} \xi_{R} d x \leq \frac{\varepsilon}{4} .
\end{aligned}
$$

This yields

$$
\begin{gathered}
\left(a+b \int_{R^{3}}\left|\nabla u_{n}\right|^{2} d x\right) \int_{R^{3}}\left|\nabla u_{n}\right|^{2} \xi_{R} d x+\int_{R^{3}}\left|u_{n}\right|^{2} \xi_{R} d x \\
+\left(a+b \int_{R^{3}}\left|\nabla u_{n}\right|^{2} d x\right) \int_{R^{3}} u_{n} \nabla u_{n} \cdot \nabla \xi_{R} d x \\
\leq \int_{R^{3}} k(x) f\left(u_{n}\right) u_{n} \xi_{R} d x+\int_{R^{3}} h(x) u_{n} \xi_{R} d x+\frac{\varepsilon}{4} .
\end{gathered}
$$

By (32), we have

$$
k(x) f\left(u_{n}\right) u_{n} \leq \theta u_{n}^{2} \quad \text { for } \theta \in(0, \min \{1, a\}),|x| \geq R_{0} .
$$

This yields

$$
\int_{R^{3}} k(x) f\left(u_{n}\right) u_{n} \xi_{R} d x \leq \theta \int_{R^{3}} u_{n}^{2} \xi_{R} d x
$$

for all $n \in N$ and $|x| \geq R_{0}$. For any $\varepsilon>0$, there exists $R(\varepsilon) \geq$ $R_{0}$ such that

$$
\frac{1}{R^{2}} \leq \frac{4 \varepsilon^{2}}{C_{4}^{2}} \quad \forall R>R(\varepsilon) .
$$

Because $h \in L^{2}\left(R^{3}\right), h \geq(\not \equiv) 0$, there exists $\bar{\rho}=\bar{\rho}(\varepsilon)$ such that

$$
\|h\|_{2, R^{3} \backslash B_{\rho}(0)}<\varepsilon, \quad \forall \rho \geq \bar{\rho} .
$$

By the Hölder inequality, (45), (55), and the boundedness of $\left\{u_{n}\right\}$ in $H^{1}\left(R^{3}\right)$, we have

$$
\begin{array}{r}
\int_{R^{3}} h(x) u_{n} \xi_{R} d x \leq\left\|h(x) \xi_{R}\right\|_{2}\left\|u_{n}\right\|_{2} \\
\leq\|h(x)\|_{2,|x|>R / 2}\left\|u_{n}\right\|_{2} \leq \frac{\varepsilon}{4} \\
\forall R>R(\varepsilon) .
\end{array}
$$


By the Young inequality, (46), and (54), for all $n \in N$ and $R>R(\varepsilon)$, we obtain

$$
\begin{aligned}
\int_{R^{3}}\left|u_{n} \nabla u_{n} \cdot \nabla \xi_{R}\right| d x \leq & \int_{R^{3}} \sqrt{2 \varepsilon}\left|\nabla u_{n}\right| \frac{1}{\sqrt{2 \varepsilon}}\left|u_{n}\right|\left|\nabla \xi_{R}\right| d x \\
\leq & \varepsilon \int_{R^{3}}\left|\nabla u_{n}\right|^{2} d x \\
& +\frac{1}{4 \varepsilon} \int_{|x| \leq R}\left|u_{n}\right|^{2} \frac{C_{4}^{2}}{R^{2}} d x \\
\leq & \varepsilon \int_{R^{3}}\left|\nabla u_{n}\right|^{2} d x \\
& +\varepsilon \int_{|x| \leq R}\left|u_{n}\right|^{2} d x \leq \varepsilon \max \{a, 1\}\left\|u_{n}\right\|^{2} .
\end{aligned}
$$

Combining $b>0$, (51), (53), (56), (57), and the boundedness of $\left\{u_{n}\right\}$ in $H^{1}\left(R^{3}\right)$, there exists $C_{6}>0$ such that

$$
\begin{aligned}
\min & \{1-\theta, 1\} \int_{R^{3}}\left(a\left|\nabla u_{n}\right|^{2}+\left|u_{n}\right|^{2}\right) \xi_{R} d x \\
& \leq \frac{\varepsilon}{2}+\varepsilon \max \{a, 1\}\left\|u_{n}\right\|^{2}\left(a+b \int_{R^{3}}\left|\nabla u_{n}\right|^{2} d x\right) \\
& \leq C_{6} \varepsilon \quad \forall R>R(\varepsilon) .
\end{aligned}
$$

Note that $C_{6}$ is independent of $\varepsilon$. So, for any $\varepsilon>0$, we can choose $R(\varepsilon)>R_{0}$ and $n(\varepsilon)>0$ such that $\int_{|x| \geq R}\left(\left|\nabla u_{n}\right|^{2}+\right.$ $\left.\left|u_{n}\right|^{2}\right) d x \leq \varepsilon$ holds.

Lemma 8. Suppose that $h \in L^{2}\left(R^{3}\right), h \geq 0$, (f1)-(f3), and $(k 1)-(k 2)$ hold. Then the sequence $\left\{u_{n}\right\}$ in (27) has a convergent subsequence. Moreover, I possesses a nonzero critical point $u$ in $H^{1}\left(R^{3}\right)$ and $I(u)>0$.

Proof. By Lemma 6, the sequence $\left\{u_{n}\right\}$ in (27) is bounded in $H^{1}\left(R^{3}\right)$. We may assume that up to a subsequence $u_{n} \rightarrow u$ weakly in $H^{1}\left(R^{3}\right)$ for some $u \in H^{1}\left(R^{3}\right)$. Now, we shall show that $\left\|u_{n}\right\| \rightarrow\|u\|$ as $n \rightarrow \infty$.

By (11), we have

$$
\begin{aligned}
\left\langle I^{\prime}\left(u_{n}\right), u_{n}\right\rangle= & \left(a+b \int_{R^{3}}\left|\nabla u_{n}\right|^{2} d x\right) \int_{R^{3}}\left|\nabla u_{n}\right|^{2} d x \\
& +\int_{R^{3}} u_{n}^{2} d x-\int_{R^{3}} k(x) f\left(u_{n}\right) u_{n} \\
& -\int_{R^{3}} h(x) u_{n} d x, \\
\left\langle I^{\prime}\left(u_{n}\right), u\right\rangle= & \left(a+b \int_{R^{3}}\left|\nabla u_{n}\right|^{2} d x\right) \int_{R^{3}} \nabla u_{n} \cdot \nabla u d x \\
& +\int_{R^{3}} u_{n} u d x-\int_{R^{3}} k(x) f\left(u_{n}\right) u \\
& -\int_{R^{3}} h(x) u d x .
\end{aligned}
$$

By (59), $b>0$, and the boundedness of $\left\{u_{n}\right\}$ in $H^{1}\left(R^{3}\right)$, we easily get

$$
\begin{aligned}
& \left\langle I^{\prime}\left(u_{n}\right), u_{n}-u\right\rangle=\left(a+b \int_{R^{3}}\left|\nabla u_{n}\right|^{2} d x\right) \int_{R^{3}}\left|\nabla u_{n}\right|^{2} d x \\
& +\int_{R^{3}} u_{n}^{2} d x-\int_{R^{3}} k(x) f\left(u_{n}\right) u_{n} d x \\
& -\int_{R^{3}} h(x) u_{n} d x \\
& -\left(a+b \int_{R^{3}}\left|\nabla u_{n}\right|^{2} d x\right) \int_{R^{3}} \nabla u \cdot \nabla u_{n} d x \\
& -\int_{R^{3}} u u_{n} d x+\int_{R^{3}} k(x) f\left(u_{n}\right) u d x \\
& +\int_{R^{3}} h(x) u d x \\
& =\left(a+b \int_{R^{3}}\left|\nabla u_{n}\right|^{2} d x\right) \\
& \times \int_{R^{3}}\left|\nabla\left(u_{n}-u\right)\right|^{2} d x \\
& +\int_{R^{3}}\left|u_{n}-u\right|^{2} d x \\
& +\left(a+b \int_{R^{3}}\left|\nabla u_{n}\right|^{2} d x\right) \\
& \times \int_{R^{3}} \nabla u \nabla\left(u_{n}-u\right) d x \\
& +\int_{R^{3}} u\left(u_{n}-u\right) d x \\
& -\int_{R^{3}} k(x) f\left(u_{n}\right)\left(u_{n}-u\right) d x \\
& -\int_{R^{3}} h(x)\left(u_{n}-u\right) d x \\
& \geq a \int_{R^{3}} \nabla\left|\left(u_{n}-u\right)\right|^{2} d x+\int_{R^{3}}\left|u_{n}-u\right|^{2} d x \\
& +\left(a+b \int_{R^{3}}\left|\nabla u_{n}\right|^{2} d x\right) \\
& \times \int_{R^{3}} \nabla u \nabla\left(u_{n}-u\right) d x \\
& +\int_{R^{3}} u\left(u_{n}-u\right) d x \\
& -\int_{R^{3}} k(x) f\left(u_{n}\right)\left(u_{n}-u\right) d x \\
& -\int_{R^{3}} h(x)\left(u_{n}-u\right) d x
\end{aligned}
$$




$$
\begin{aligned}
& \geq\left\|u_{n}-u\right\|^{2}+\left(a+b \int_{R^{3}}\left|\nabla u_{n}\right|^{2} d x\right) \\
& \quad \times \int_{R^{3}} \nabla u \nabla\left(u_{n}-u\right) d x \\
& +\int_{R^{3}} u\left(u_{n}-u\right) d x \\
& -\int_{R^{3}} k(x) f\left(u_{n}\right)\left(u_{n}-u\right) d x \\
& -\int_{R^{3}} h(x)\left(u_{n}-u\right) d x .
\end{aligned}
$$

One has

$$
\begin{aligned}
\left\|u_{n}-u\right\|^{2} \leq & \left\langle I^{\prime}\left(u_{n}\right), u_{n}-u\right\rangle \\
& -\left(a+b \int_{R^{3}}\left|\nabla u_{n}\right|^{2} d x\right) \int_{R^{3}} \nabla u \nabla\left(u_{n}-u\right) d x \\
& -\int_{R^{3}} u\left(u_{n}-u\right) d x \\
& +\int_{R^{3}} k(x) f\left(u_{n}\right)\left(u_{n}-u\right) d x \\
& +\int_{R^{3}} h(x)\left(u_{n}-u\right) d x .
\end{aligned}
$$

It is clear that

$$
\left\langle I^{\prime}\left(u_{n}\right), u_{n}-u\right\rangle \longrightarrow 0, \quad \text { as } n \longrightarrow \infty
$$

Since $u_{n} \rightarrow u$ weakly in $H^{1}\left(R^{3}\right)$, we obtain

$$
\begin{aligned}
\int_{R^{3}}\left(\nabla u_{n} \cdot \nabla u+u_{n} u\right) d x= & \int_{R^{3}}\left(|\nabla u|^{2}+|u|^{2}\right) d x \\
& +o(1), \quad \text { as } n \longrightarrow \infty .
\end{aligned}
$$

By the continuity of imbedding $H^{1}\left(R^{3}\right) \hookrightarrow L^{2}\left(R^{3}\right)$, we have that $u_{n} \rightarrow u$ weakly in $L^{2}\left(R^{3}\right)$; that is,

$$
\int_{R^{3}} u_{n} u d x=\int_{R^{3}} u^{2} d x+o(1), \quad \text { as } n \longrightarrow \infty
$$

By (63) and (64), we deduce

$$
\int_{R^{3}} \nabla u_{n} \cdot \nabla u d x=\int_{R^{3}}|\nabla u|^{2} d x+o(1), \quad \text { as } n \longrightarrow \infty .
$$

Combining the boundedness of $\left\{u_{n}\right\}$ in $H^{1}\left(R^{3}\right),(64)$, and (65), we obtain

$$
\begin{aligned}
\left(a+b \int_{R^{3}}\left|\nabla u_{n}\right|^{2} d x\right) \int_{R^{3}} \nabla u \nabla\left(u_{n}-u\right) d x \\
\quad+\int_{R^{3}} u\left(u_{n}-u\right) d x=o(1), \quad \text { as } n \longrightarrow \infty .
\end{aligned}
$$

Moreover, by (32), Lemma 7, and $u_{n} \rightarrow u$ in $H^{1}\left(R^{3}\right)$, for any $\varepsilon>0$ and $n$ large enough, one has

$$
\begin{aligned}
\int_{|x| \geq R(\varepsilon)} k(x) f\left(u_{n}\right) u_{n} d x-\int_{|x| \geq R(\varepsilon)} k(x) f\left(u_{n}\right) u d x \\
=\int_{|x| \geq R(\varepsilon)} k(x) f\left(u_{n}\right)\left(u_{n}-u\right) d x \\
\leq \int_{|x| \geq R(\varepsilon)}\left|k(x) f\left(u_{n}\right)\right|\left|u_{n}-u\right| d x \\
\leq\left(\int_{|x| \geq R(\varepsilon)}\left|k^{2}(x) f^{2}\left(u_{n}\right)\right| d x\right)^{1 / 2} \\
\quad \times\left(\int_{|x| \geq R(\varepsilon)}\left|u_{n}-u\right|^{2} d x\right)^{1 / 2} \\
\leq \theta\left(\int_{|x| \geq R(\varepsilon)}\left|u_{n}^{2}\right| d x\right)^{1 / 2}\left(\int_{|x| \geq R(\varepsilon)}\left|u_{n}-u\right|^{2} d x\right)^{1 / 2} \\
\leq \theta\left(\int_{|x| \geq R(\varepsilon)}\left(a\left|\nabla u_{n}\right|^{2}+\left|u_{n}\right|^{2}\right) d x\right)^{1 / 2} \\
\quad \times\left(\int_{|x| \geq R(\varepsilon)}\left|u_{n}-u\right|^{2} d x\right)^{1 / 2} \leq \theta \varepsilon .
\end{aligned}
$$

This and the compactness of embedding $H^{1}\left(R^{3}\right) \hookrightarrow L_{\text {loc }}^{2}\left(R^{3}\right)$ imply that

$$
\int_{R^{3}} k(x) f\left(u_{n}\right) u_{n} d x=\int_{R^{3}} k(x) f\left(u_{n}\right) u d x+o(1) .
$$

Since $u_{n}$ is bounded in $H^{1}\left(R^{3}\right)$ and the continuity of the Sobolev embedding of $H^{1}\left(R^{3}\right)$ imbedding in $L^{2}\left(R^{3}\right)$, for any choice of $\varepsilon>0$ and $\rho>0$, the relation

$$
\left\|u_{n}-u\right\|_{2, B_{\rho}(0)}<\varepsilon
$$

holds for large $n$. By $h \in L^{2}\left(R^{3}\right)$, for any $\varepsilon>0$ there exists $\bar{\rho}=\bar{\rho}(\varepsilon)$ such that

$$
\|h\|_{2, R^{3} \backslash B_{\rho}(0)}<\varepsilon, \quad \forall \rho \geq \bar{\rho} .
$$

By (70) and (69), we have

$$
\begin{aligned}
\int_{R^{3}} h(x) u_{n} d x-\int_{R^{3}} h(x) u d x \\
\leq \int_{R^{3} \backslash B_{\rho}(0)}\left|h(x)\left(u_{n}-u\right)\right| d x \\
\quad+\int_{B_{\rho}(0)}\left|h(x)\left(u_{n}-u\right)\right| d x \\
\leq\|h(x)\|_{2, R^{3} \backslash B_{\rho}(0)}\left\|u_{n}-u\right\|_{2, R^{3} \backslash B_{\rho}(0)} \\
\quad+\|h(x)\|_{2, B_{\rho}(0)}\left\|u_{n}-u\right\|_{2, B_{\rho}(0)} \\
\leq \varepsilon\left\|u_{n}-u\right\|_{2, R^{3} \backslash B_{\rho}(0)}+\varepsilon\|h(x)\|_{2, B_{\rho}(0)} \\
\leq C_{7} \varepsilon .
\end{aligned}
$$


This yields

$$
\int_{R^{3}} h(x) u_{n} d x=\int_{R^{3}} h(x) u d x+o(1) .
$$

By (61), (62), (66), (68), and (72), we have

$$
\left\|u_{n}-u\right\|^{2}=o(1), \quad \text { as } n \longrightarrow \infty,
$$

by $a>0$. This yields that $\left\|u_{n}\right\| \rightarrow\|u\|$ as $n \rightarrow \infty$ and $u$ is a nonzero critical point of $I$ in $H^{1}\left(R^{3}\right)$ and $I(u)>0$ by Mountain Pass Theorem in [35].

Now, we give local properties of the variational functional $I$, which is required by using Ekeland's variational principle.

Lemma 9. Suppose that $h \in L^{2}\left(R^{3}\right), h \geq(\not \equiv) 0,(f 1)-(f 3)$, and (k1) hold. If $\|h\|_{2}<m$, then there exists $u_{0} \in H^{1}\left(R^{3}\right)$ such that

$$
\begin{aligned}
& I\left(u_{0}\right)=\inf \left\{I(u): u \in \bar{B}_{\rho}\right\}<0, \\
& \text { where } B_{\rho}=\left\{u \in H^{1}\left(R^{3}\right):\|u\|<\rho\right\},
\end{aligned}
$$

$m, \rho$ are given by Lemma 4 and $u_{0}$ is a positive solution of system (1).

Proof. Because $h \in L^{2}\left(R^{3}\right), h \geq(\not \equiv) 0$, we can choose a function $\varphi \in H^{1}\left(R^{3}\right)$ such that

$$
\int_{R^{3}} h(x) \varphi d x>0 .
$$

Together with (f1), (k1), and (75), for $t>0$, we have

$$
\begin{aligned}
I(t \varphi)= & \frac{a t^{2}}{2} \int_{R^{3}}|\nabla \varphi|^{2} d x+\frac{b t^{4}}{4}\left(\int_{R^{3}}|\nabla \varphi|^{2} d x\right)^{2} \\
& +\frac{t^{2}}{2} \int_{R^{3}}|\varphi|^{2} d x \\
& -\int_{R^{3}} k(x) F(t \varphi) d x-\int_{R^{3}} h(x) t \varphi d x \\
\leq & \frac{t^{2}}{2}\|\varphi\|^{2}+\frac{b t^{4}}{4}\|\varphi\|^{4}-t \int_{R^{3}} h(x) \varphi d x \\
\leq & 0
\end{aligned}
$$

for $t>0$ small enough. Thus there exists $u$ small enough such that $I(u)<0$. By Lemma 4 , we deduce that

$$
c_{0}:=\inf _{u \in \bar{B}_{\rho}} I(u)<0<\inf _{u \in \partial \bar{B}_{\rho}} I(u) .
$$

By applying Ekeland's variational principle [36, Theorem 4.1] in $\bar{B}_{\rho}$, there is a minimizing sequence $\left\{u_{n}\right\} \subset \bar{B}_{\rho}$ such that

$$
\begin{aligned}
& \text { (i) } c_{0} \leq I\left(u_{n}\right)<c_{0}+\frac{1}{n}, \\
& \text { (ii) } I(w) \geq I\left(u_{n}\right)-\frac{1}{n}\left\|w-u_{n}\right\| \quad \forall w \in \bar{B}_{\rho} .
\end{aligned}
$$

Then, by a standard procedure, we can show that $\left\{u_{n}\right\}$ is a bounded (PS) sequence of $I$. Lemmas 7 and 8 imply that there exists $u_{0} \in H^{1}\left(R^{3}\right)$ such that $I^{\prime}\left(u_{0}\right)=0$ and $I\left(u_{0}\right)=c_{0}<0$. So this lemma is proved.
Proof of Theorem 1.2. By Lemmas 4-8, we obtain the existence of a mountain pass solution $u$ for problem (1) and $I(u)>0$. By Lemma 9, we know that problem (1) has a local minimum solution $u_{0}$ and $I\left(u_{0}\right)<0$. Thus, $u \neq u_{0}$ and $u, u_{0}$ are positive. Thus this theorem is proved.

\section{Conflict of Interests}

The authors declare that there is no conflict of interests regarding the publication of this paper.

\section{Acknowledgments}

The authors express their thanks to Professor Chun-Lei Tang for his valuable help and suggestion during their stay in Southwest University. The authors would like to thank the referee for valuable suggestions. The first author was supported by the National Natural Science Foundation of China (no. 11101347), Postdoctor Foundation of China (no. 2012M510363) and the Key Project in Science and Technology Research Plan of the Education Department of Hubei Province (nos. D20112605 and D20122501), while the second one by the National Natural Science Foundation of China (no. 11201323) and the Fundamental Research Funds for the Central Universities (no. XDJK2013D007).

\section{References}

[1] G. R. Kirchhoff, Vorlesungen Über Mathematische Physik, Teubner, Leipzig, Germany, 1883.

[2] C. O. Alves, F. J. S. A. Corrêa, and T. F. Ma, "Positive solutions for a quasilinear elliptic equation of Kirchhoff type," Computers and Mathematics with Applications, vol. 49, no. 1, pp. 85-93, 2005.

[3] S. Bernstein, "Sur une classe d'equations fonctionnelles aux derivees," Bulletin of the Academy of Sciences of the USSR Series, vol. 4, no. 1, pp. 17-26, 1940.

[4] S. I. Pohozaev, "A certain class of quasilinear hyperbolic equations," Matematicheskii Sbornik, vol. 96, no. 138, pp. 152-166, 168, 1975 (Russian).

[5] J. L. Lions, "On some questions in boundary value problems of mathematical physics. Contemporary developments in continuum mechanics and partial differential equations," in Proceedings of the International Symposium, Instituto de Matemática, Universidade Federal do Rio de Janeiro, Rio De Janeiro, Brazil, 1977, North-Holland Mathematics Studies, pp. 284-346, NorthHolland Publishing, Amsterdam, The Netherlands, 1978.

[6] A. Arosio and S. Panizzi, "On the well-posedness of the Kirchhoff string," Transactions of the American Mathematical Society, vol. 348, no. 1, pp. 305-330, 1996.

[7] M. M. Cavalcanti, V. N. D. Cavalcanti, and J. A. Soriano, "Global existence and uniform decay rates for the Kirchhoff-Carrier equation with nonlinear dissipation," Advances in Differential Equations, vol. 6, no. 6, pp. 701-730, 2001.

[8] P. D'Ancona and S. Spagnolo, "Global solvability for the degenerate Kirchhoff equation with real analytic data," Inventiones Mathematicae, vol. 108, no. 1, pp. 247-262, 1992.

[9] B. Cheng and X. Wu, "Existence results of positive solutions of Kirchhoff type problems," Nonlinear Analysis: Theory, Methods and Applications, vol. 71, no. 10, pp. 4883-4892, 2009. 
[10] X. He and W. Zou, "Infinitely many positive solutions for Kirchhoff-type problems," Nonlinear Analysis: Theory, Methods and Applications, vol. 70, no. 3, pp. 1407-1414, 2009.

[11] T. F. Ma and J. E. Muñoz Rivera, "Positive solutions for a nonlinear nonlocal elliptic transmission problem," Applied Mathematics Letters, vol. 16, no. 2, pp. 243-248, 2003.

[12] A. Mao and Z. Zhang, "Sign-changing and multiple solutions of Kirchhoff type problems without the P.S. condition," Nonlinear Analysis: Theory, Methods and Applications, vol. 70, no. 3, pp. 1275-1287, 2009.

[13] K. Perera and Z. Zhang, "Nontrivial solutions of Kirchhoff-type problems via the Yang index," Journal of Differential Equations, vol. 221, no. 1, pp. 246-255, 2006.

[14] Z. Zhang and K. Perera, "Sign changing solutions of Kirchhoff type problems via invariant sets of descent flow," Journal of Mathematical Analysis and Applications, vol. 317, no. 2, pp. 456463, 2006.

[15] X. He and W. Zou, "Multiplicity of solutions for a class of Kirchhoff type problems," Acta Mathematicae Applicatae Sinica, vol. 26, no. 3, pp. 387-394, 2010.

[16] Y. Yang and J. Zhang, "Positive and negative solutions of a class of nonlocal problems," Nonlinear Analysis: Theory, Methods and Applications, vol. 73, no. 1, pp. 25-30, 2010.

[17] Y. Yang and J. Zhang, "Nontrivial solutions of a class of nonlocal problems via local linking theory," Applied Mathematics Letters, vol. 23, no. 4, pp. 377-380, 2010.

[18] C. Chen, Y. Kuo, and T. Wu, "The Nehari manifold for a Kirchhoff type problem involving sign-changing weight functions," Journal of Differential Equations, vol. 250, no. 4, pp. 1876-1908, 2011.

[19] J. Sun and C. Tang, "Existence and multiplicity of solutions for Kirchhoff type equations," Nonlinear Analysis: Theory, Methods and Applications, vol. 74, no. 4, pp. 1212-1222, 2011.

[20] J. Sun and S. Liu, "Nontrivial solutions of Kirchhoff type problems," Applied Mathematics Letters, vol. 25, no. 3, pp. 500504, 2012.

[21] A. Mao and S. Luan, "Sign-changing solutions of a class of nonlocal quasilinear elliptic boundary value problems," Journal of Mathematical Analysis and Applications, vol. 383, no. 1, pp. 239-243, 2011.

[22] B. T. Cheng, "New existence and multiplicity of nontrivial solutions for nonlocal elliptic Kirchhoff type problems," Journal of Mathematical Analysis and Applications, vol. 394, no. 2, pp. 488-495, 2012.

[23] S. J. Chen and L. Li, "Multiple solutions for the nonhomogeneous Kirchhoff equation on RN," Nonliear Analysis: Real World Applications, vol. 14, pp. 1477-1486, 2012.

[24] J. Jin and X. Wu, "Infinitely many radial solutions for Kirchhofftype problems in $R^{N}$," Journal of Mathematical Analysis and Applications, vol. 369, no. 2, pp. 564-574, 2010.

[25] X. Wu, "Existence of nontrivial solutions and high energy solutions for Schrödinger-Kirchhoff-type equations in $R^{N}$," Nonlinear Analysis: Real World Applications, vol. 12, no. 2, pp. 1278-1287, 2011.

[26] A. Azzollini, P. d’Avenia, and A. Pomponio, "Multiple critical points for a class of nonlinear functionals," Annali di Matematica Pura ed Applicata, vol. 190, no. 3, pp. 507-523, 2011.

[27] H. Berestycki and P.-L. Lions, "Nonlinear scalar field equations. II: existence of infinitely many solutions," Archive for Rational Mechanics and Analysis, vol. 82, no. 4, pp. 347-375, 1983.
[28] X. He and W. Zou, "Existence and concentration behavior of positive solutions for a Kirchhoff equation in $R^{3}$," Journal of Differential Equations, vol. 252, no. 2, pp. 1813-1834, 2012.

[29] J. Nie and X. Wu, "Existence and multiplicity of non-trivial solutions for SchrödingerKirchhoff-type equations with radial potential," Nonlinear Analysis: Theory, Methods and Applications, vol. 75, no. 8, pp. 3470-3479, 2012.

[30] C. O. Alves and G. M. Figueiredo, "Nonlinear perturbations of a periodic Kirchhoff equation in $R^{N}$," Nonlinear Analysis: Theory, Methods and Applications, vol. 75, no. 5, pp. 2750-2759, 2012.

[31] W. Liu and X. He, "Multiplicity of high energy solutions for superlinear Kirchhoff equations," Journal of Applied Mathematics and Computing, vol. 39, no. 1-2, pp. 473-487, 2012.

[32] J. Sun, H. Chen, and J. J. Nieto, "On ground state solutions for some non-autonomous Schrödinger-Poisson systems," Journal of Differential Equations, vol. 252, no. 5, pp. 3365-3380, 2012.

[33] Z. Wang and H. Zhou, "Positive solutions for a nonhomogeneous elliptic equation on $R^{N}$ without $(A R)$ condition," Journal of Mathematical Analysis and Applications, vol. 353, no. 1, pp. 470-479, 2009.

[34] C. Liu, Z. Wang, and H. Zhou, "Asymptotically linear Schrödinger equation with potential vanishing at infinity," Journal of Differential Equations, vol. 245, no. 1, pp. 201-222, 2008.

[35] I. Ekeland, "On the variational principle," Journal of Mathematical Analysis and Applications, vol. 47, no. 2, pp. 324-353, 1974.

[36] J. Mawhin and M. Willem, Critical Point Theory and Hamiltonian Systems, vol. 74 of Applied Mathematical Sciences, Springer, New York, NY, USA, 1989. 


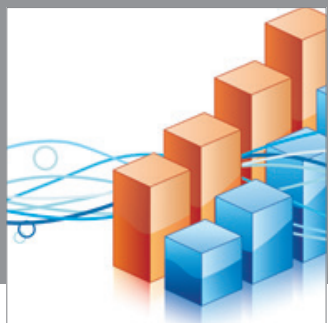

Advances in

Operations Research

mansans

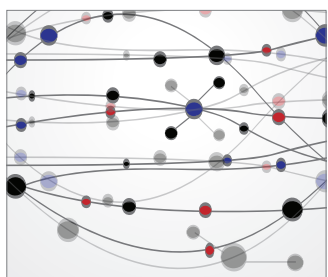

The Scientific World Journal
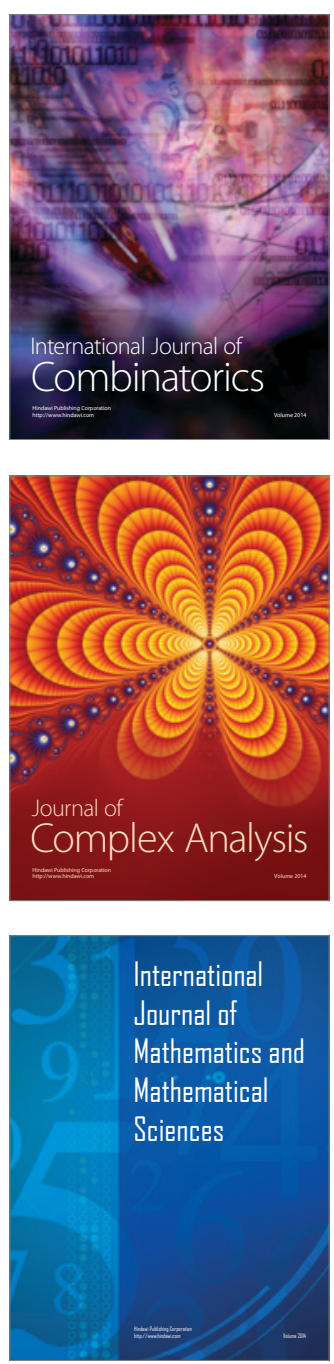
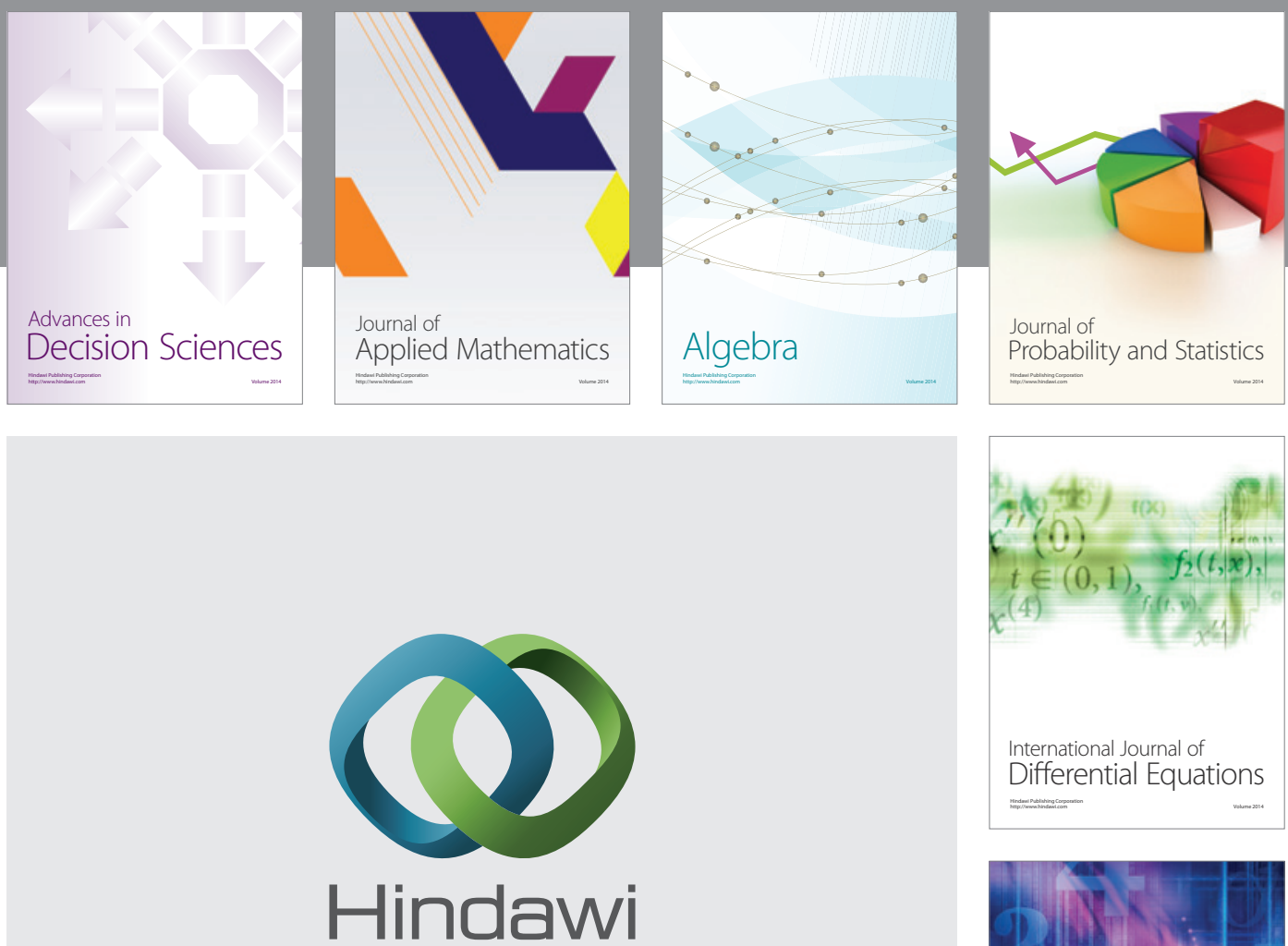

Submit your manuscripts at http://www.hindawi.com
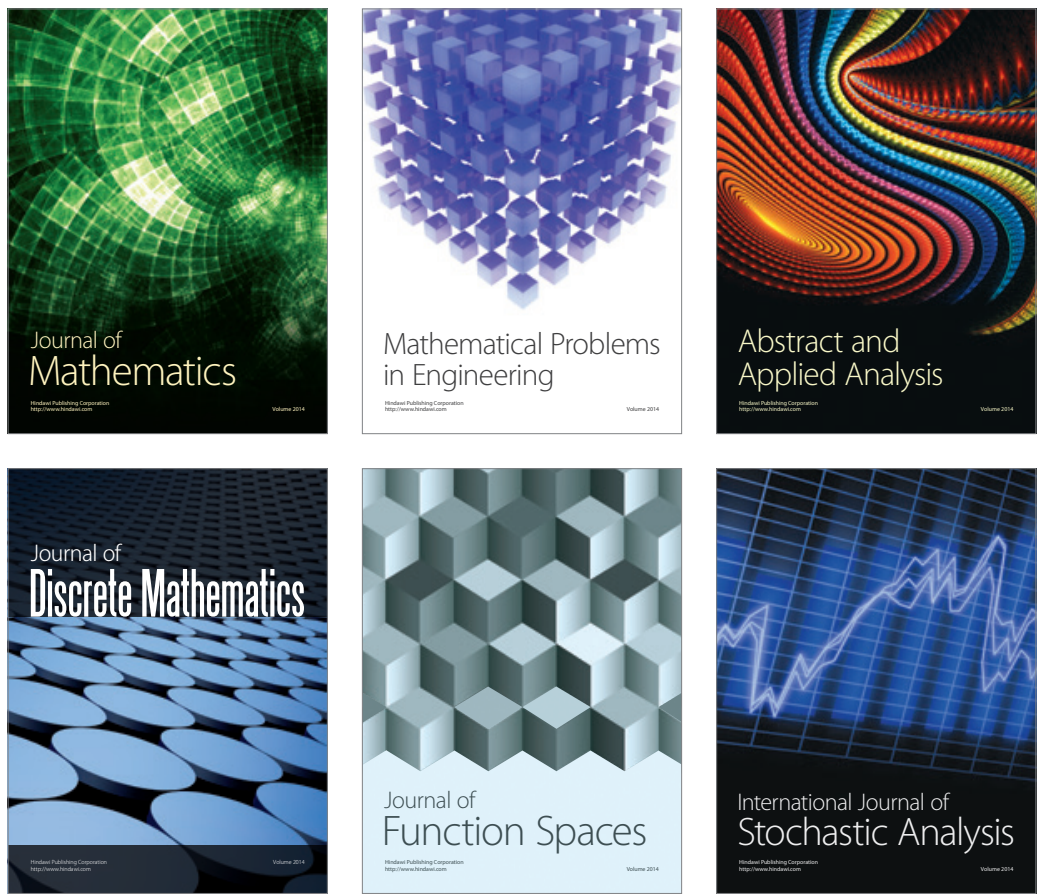

Journal of

Function Spaces

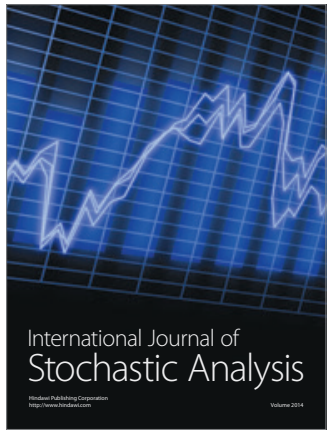

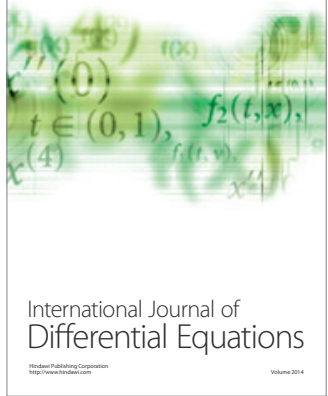
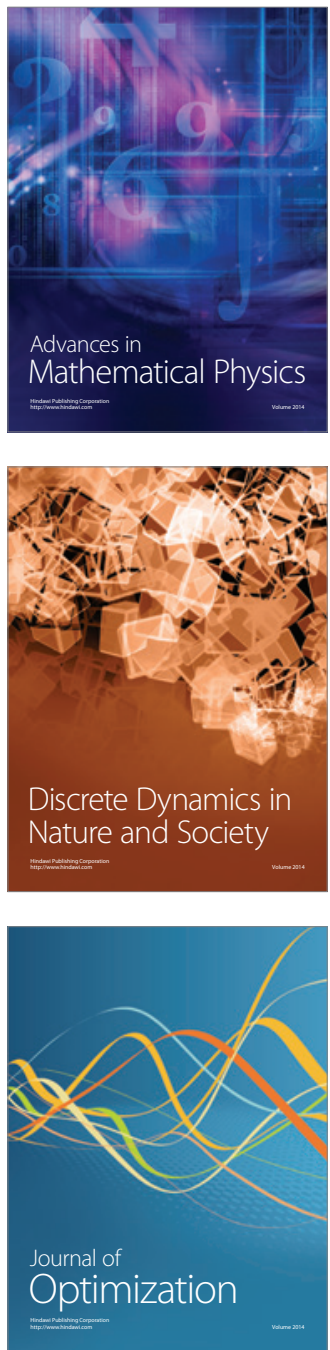